\title{
A Conversation with Monica Colaiácovo
}

\author{
INTERVIEWER: LARA SZEWCZAK \\ Scientific Editor, Cell
}

\begin{abstract}
Monica Colaiácovo is a Professor in the Department of Genetics at Harvard Medical
School, Boston, Massachusetts.
\end{abstract}

\begin{abstract}
Lara Szewczak: You work on meiosis, in particular on the process of sorting out where crossovers happen. Maybe we can just start with a brief explanation of what that actually means.
\end{abstract}

Dr. Colaiácovo: Crossovers are extremely important because they provide a way to exchange genetic information between the chromosomes during meiosis. That means that it provides genetic diversity. But also, in the context of meiosis it creates a locked-in interaction between the pairs of homologous chromosomes so that, as they progress and finally align at the metaphase plate and microtubules attach, you'll have enough tension there to ensure that the chromosomes will segregate away from each other appropriately to opposite ends of the spindle. It is important to get that crossover. It has to be tightly regulated. It is incredibly important for successful and accurate segregation of the chromosomes during meiosis.

It is very clear that crossovers are tightly regulated. They're not randomly distributed, and that's universal. We see that in mammals, we see it in C. elegans-the model organism we use in my lab - and budding yeast, and plants, and flies. There is always an obligate crossover, which means that there's a push in the system so that you will always get at least one crossover per homolog pair to ensure that there'll be that attachment.

There is also the phenomenon of crossover interference. Although chromosomes are undergoing multiple programmed meiotic double-strand breaks (which is a unique feature of meiosis; you actually want to make those breaks so that you will engage into a crossover outcome), only a subset of those breaks will then get designated or destined to become a crossover. Once one is selected to become a crossover, other breaks nearby are not repaired as a crossover event; that single crossover interferes with the probability of getting crossovers nearby.

All these different layers of regulation told us this is a system that's very robust. It has to be tightly regulated, but why is it that the position of the crossover might matter? We were intrigued by that question because there's a lot of information from human studies showing an association with aneuploidy in trisomy 21 , as well as the majority of examples of trisomy 16 , with the position of the crossover.
If it's very terminally located, close to the end of a chromosome, it doesn't sustain normal or accurate chromosome segregation. Why is that?

We decided to look at that in a metazoan, because that had not been directly tested in a metazoan system before. To do that, we co-opted the power of transposons. It turns out that there is a Mariner transposon that was imported into C. elegans from Drosophila; this was beautiful work done by Erik Jorgensen and [Jean-Louis] Bessereau's lab. Basically, they generated these lines where you would have a single transposon insertion at a discrete place in the genome. We screened many of these lines to find specific ones where we knew the transposon was excised at a very high frequency in an efficient manner. The way these are excised is because you have a transposase under a heat shock promoter. To make sure that the only source of the break was going to be the system that we were going to be using, the inducible system, we crossed that into a spo-11 mutant background where you no longer have the programmed formation of meiotic double-strand breaks.

Now we could look at a break generated at a specific location and ask the question: "When that break gets resolved as a crossover, what is going on?" Is it really going to succeed in generating a functional attachment between the homologs that will facilitate the accurate segregation of the homologs away from each other? What happens if we make a break right at the physical center of a chromosome, or right at the end, which, anecdotally, should be problematic...

\section{Lara Szewczak: So, telomeric?}

Dr. Colaiácovo: Subtelomeric, and then what would happen if it happened in an off-centered position, which is normally where crossovers happen in C. elegans, because that's an important control. It would tell us whether the Mos system, this transposon system, was working as a normal SPO-11 break might. What we found was that the control of an off-centered event is productive. It results in a functional chiasma and all the chromosome remodeling that has to happen after the crossover proceeds in a normal fashion. But if the break that got selected to become a crossover was a break right in the physical center

(C) 2017 Colaiácovo. This article is distributed under the terms of the Creative Commons Attribution-NonCommercial License, which permits reuse and redistribution, except for commercial purposes, provided that the original author and source are credited. 
of a chromosome, that did not succeed in sustaining an accurate attachment between the chromosomes, although we would still see the ability to form a cruciform structure.

What that means is: you've got the break. It becomes a crossover, and what happens next is you're going to have massive remodeling of these chromosomes. You're going to lose some proteins and gain other novel proteins at very specific chromosomal subdomains. Ultimately, there's also increased compaction of the chromosomes. What you acquire is a very characteristic configuration. It actually looks like a cruciform with the short and long axes, and there's a reason for that. Because of how chromosomes have to be aligned at the metaphase plate, the short arms are aligned equatorially; the long arms face the opposite spindles. What you want is to remove cohesin at that interface between the short arms so that now the homologs can segregate away from each other.

We examined if that process of remodeling occurs normally. We found that when that break is in the middle and a crossover is the outcome of that, you still formed a cruciform, but it was no longer an asymmetric cruciform. It was now a symmetric bivalent, and some of the proteins that should have been retained at a specific subdomain were no longer there. These proteins... in this case, LAB-1, which is a functional ortholog of Shugoshin and plays a very important role in insulating a specific domain (the long arms) from premature loss of sister chromatid cohesion, that domain was no longer protected. Now you had a mislocalization of Aurora B kinase to both the short and the long arms of the bivalent.

So we took it a step further, and monitored the metaphase-to-anaphase transition to see whether that mislocalization indeed resulted in premature loss of sister chromatid cohesion, and it does. We concluded that maybe part of the reason you don't normally use breaks at the center region of chromosomes to become a crossover is that you won't be able to sustain normal remodeling. You'll result in a bivalent, but that attachment is no longer productive, in the sense that it can't promote accurate chromosome segregation. That was very interesting, because it gave us a more logical idea of the basic mechanism as to why there's a disconnect between [the fact that there is the formation of] all these breaks, but you tend to select certain ones and not others [as crossovers].

Then we asked the question, "Well, what happens if this is now located at a subtelomeric region?" We've looked at a lot of events, a lot of oocytes, and we cannot find a bivalent at the end of diakinesis. Remember, this is a pipeline. We can see that we succeeded in making a break at the subtelomeric end; we've got markers for that. We know that a subset of those is getting selected to undergo a crossover path because we do see procrossover markers at that region, but we can't see an actual chiasma at the end of diakinesis. This tells us that these subtelomeric events are incredibly unable to actually retain that attachment. Something is sliding off; something is not working properly there. It is very analogous to what we think goes on in mammalian systems where, if you've got these events that are designated to become a crossover, it doesn't really result in an effective, functional, accurate segregation event.
Lara Szewczak: Is one way to think about it is that if you're subtelomeric, there's just not enough DNA to work with?

Dr. Colaiácovo: That's one possibility.

Lara Szewczak: How does the measuring work? How do you know where you are, and does that have to do with crossover interference?

Dr. Colaiácovo: We hypothesize that there has to be a way for you to be measuring distances. We did a screen to try to understand how that might work where we took advantage of Aurora B kinase fused to GFP [green fluorescence protein]. We looked at genes that, when depleted by RNAi, would interfere with the ability of Aurora B kinase to localize only on one but not the other arm. We found a lot of interesting candidates where either Aurora B kinase cannot localize at all on these bivalents, or completely mislocalizes and is now in both axes. We focused on the subset with Aurora B kinase on both axes and we noticed that a related set of proteins was coming up. These genes encode for a specific set of proteins, all of which tell us that there has to be a way for you to communicate with telomere ends: these are telomere-associated factors. That's one end of the equation. That could be one way you're communicating this. We're currently testing that hypothesis.

The other question we had was, "Are there any other features?" There could be DNA topology that's playing a role here. There could be issues about the chromatin landscape and epigenetic readouts: Are you reading differences between one direction versus the other direction? We've already been testing this using the inducible system. We found that if the break that gets selected to become a crossover is right at the middle of a chromosome (versus an offcenter choice), before the formation of that break and then after the formation of that break, the marks that we see in both directions - the histone methyl marks and acetyl marks - toward the chromosome ends are not the same. When you have an event that happens at the physical middle, the changes throughout the full length of these chromosomes are very different from the changes we see when it's an off-centered event. Something isn't working properly when you've got that selection right at the middle.

Lara Szewczak: When you have an off-center event, are the marks on either side different, and when you make it in the middle, do the two sides look like one or the other of the off-center?

Dr. Colaiácovo: There's a difference when it's an offcentered event, which makes sense, because that could be part of how you're reading that distance. That difference is gone if it's in the middle. We're trying to figure out if there is a problem with who gets recruited that might be working as specific methyltransferases and so on that regulate this process. Is that part of why you get miscommunication, an inability to signal or read distances toward one end? How that gets superimposed onto these telomereassociated proteins, we don't know yet. We're trying to figure out how these two might be interfacing. 
Lara Szewczak: I'm just trying to understand this one point. When you have an event in the middle and you get different marks, does it look like one side of the offcenter? Is it that it can no longer distinguish one side from the other, or is it completely different from the two sides?

Dr. Colaiácovo: It can no longer distinguish one side from the other. It just looks the same in both directions, as opposed to one kind of mark acquisition in one direction versus not having that on the other.

Lara Szewczak: You've done this in worms and want to extrapolate to mammalian cells, our cells. What are the differences between the two systems that you're going to have to account for?

Dr. Colaiácovo: In C. elegans you're dealing with holocentric chromosomes. We don't have a discretely positioned centromere. In C. elegans chromosomes, you're incredibly dependent on this remodeling, because it then allows you to bypass the fact that you don't have a localized centromere to promote that proper positioning, attachment, tension, and so on. You have to be really precise with the remodeling in $C$. elegans, because that will dictate a lot of how precisely you'll be able to segregate in the metaphase-to-anaphase transition.

Having said that, the chromosome remodeling observation is not unique to worms. It's been demonstrated in flies, in yeast, and in mammals. We know that when the synaptonemal complex [SC] disassembles - it's one of the other features associated with this remodeling process - it does so in an asymmetric manner. It does so in the same way in these other systems, except that there you retain those SC proteins on the centromere. It makes sense that, in our case, they get retained on the short arm, particularly if we think about what allows you to properly align at the metaphase plate and where there's a lot of microtubule polymerization that then allows you to push these chromosomes apart from each other; it behaves a lot like a centromere. Work from Arshad Desai's lab supports that; he thinks there's good assembly and enrichment for certain factors that promote segregation in terms of kinetochore activity and so on. Although $C$. elegans may be more reliant on this because it is key for it to achieve accurate segregation, I don't think this is going to be restricted to worms, because the remodeling is pretty universal.
The beauty of this is that, if you think about what we already know about aneuploidy in humans, this directly correlates with what we see now in a testable setup in the context of what we can do in the worm.

Lara Szewczak: You talked about figuring out this measuring mechanism and unpacking potential for communication to the telomere. Thinking beyond that, what's the next question you want to ask?

Dr. Colaiácovo: We are trying to understand how that scaffold, the zipper-like synaptonemal complex, actually deals with all of these changes. How do you regulate this so that it knows where to go in an asymmetric manner, and what is the triggering point for this? That's one of the main directions we're headed: trying to focus more on the synaptonemal complex as another key element here.

Lara Szewczak: Are there particular technologies that are going to help you do that?

Dr. Colaiácovo: We started looking a lot at chromosome dynamics, so we've done a lot of FRAP [fluorescence recovery after photobleaching] experiments and measuring the ability to repopulate certain subdomains when they're depleted of a certain protein. That gives us important information about how dynamic or not these arms are before or after certain key events during meiosis. In addition, we are now doing a lot of superresolution imaging. In these cruciform structures in particular, we're going to be able to see a lot of information that - even with high-resolution microscopy — we are missing. That will hopefully allow us to tease apart more of the details of what's going on with the remodeling.

Lara Szewczak: What's the one thing that you're taking away from this meeting that is new information or a new way of thinking about your own work that's going to influence what you do?

Dr. Colaiácovo: The concept of how we can integrate a lot of what we're seeing with really beautiful 3D modeling. The concept of whether something is a gel, or a liquid. I think all of that is fascinating and is not normally the way we think about things or the way we present our information, and that's definitely something that I look forward to incorporating into what we do. 


\section{$\$_{\text {CSH\& }}^{\infty}$ Cold Spring Harbor Symposia SYMPOSIA}

\section{A Conversation with Monica Colaiácovo}

Cold Spring Harb Symp Quant Biol 2017 82: 381-383 originally published online March 26, 2018 Access the most recent version at doi:10.1101/sqb.2017.82.034462

Creative This article is distributed under the terms of the

Commons http://creativecommons.org/licenses/by-nc/4.0/, which permits reuse and

License redistribution, except for commercial purposes, provided that the original author and source are credited.

Email Alerting Receive free email alerts when new articles cite this article - sign up in Service the box at the top right corner of the article or click here. 